\title{
Allergic BronchoPulmonary Aspergillosis in Nepal
}

\author{
Neha Agarwal, 'Prajowl Shrestha, ${ }^{1}$ Ramesh Chokhani' \\ 'Department of Pulmonary Medicine, Norvic International Hospital, Kathmandu, Nepal.
}

\section{ABSTRACT}

Allergic BronchoPulmonary Aspergillosis (ABPA) is clinically under recognized and often misdiagnosed in Nepal. A total of 15 cases of ABPA were enrolled in this case series. Cases were followed up after the 3 months of start of treatment and clinical responses were assessed. The mean age of the patients was $33.06 \pm 9.2$ yrs. $80 \%$ were male. $26.6 \%$ patients were empirically on antitubercular treatment at the time of presentation. $40 \%$ patients were misdiagnosed and already treated as pulmonary Tuberculosis. 10 patients were previously treated as cases of refractory asthma. Majority of patients had significant eosinophillia and raised total serum IgE. All patients required treatment with bronchodilator and systemic steroid. $80 \%$ patients underwent remission with the treatment. Pulmonary Tuberculosis and refractory bronchial asthma are common condition that leads to the misdiagnosis of this disease.

Keywords: ABPA; bronchial asthma; central bronchiectasis; eosinophil count; serum IgE.

\section{INTRODUCTION}

Allergic BronchoPulmonary Aspergillosis (ABPA) is a pulmonary disorder caused by immunologic reactions to antigens released by Aspergillus fumigatus, colonizing the airways of patients with asthma and cystic fibrosis. ${ }^{1}$ Clinically, ABPA presents with uncontrolled asthma, expectoration of mucus plugs, fleeting pulmonary opacities and bronchiectasis. ${ }^{2,3}$

The diagnosis of ABPA is currently made on the basis of Rosenberg Patterson criteria which is combination of clinical, radiological and immunological findings. ${ }^{4,5}$

ABPA is clinically under recognized and often misdiagnosed in Nepal. The main objective of this study is to highlight the some salient clinical features, diagnosis and treatment together with some demographic profile of this disease in our context.

\section{METHODS}

A total of 15 cases of ABPA were enrolled. Cases were enrolled both retrospectively and prospectively from the day 1 of study period. Rosenberg and Patterson criterion was used for the diagnosis of ABPA. Prospective cases were followed up after the 3 months of start of treatment and clinical response was assessed.

The mean age of the patients was $33.06 \pm 9.2 \mathrm{yrs}$ (Mean \pm SD). 12 patients $(80 \%)$ were male and 3 patients $(20 \%)$ were female. Clinical characteristics of patients are outlined in Table no. 1. Cough and fever was the most common presenting clinical feature. Classically described brownish colored sputum was

Correspondence: Dr Ramesh Chokhani, Norvic International Hospital, Thapathali, Kathmandu, Nepal. Email: ramesh_chokhani@ yahoo.co.in, Phone: $+977-9851043632$ 
Agarwal et al. Allergic BronchoPulmonary Aspergillosis in Nepal

present in $80 \%$ of patients. $53.3 \%$ of patients also had hemoptysis at the time of presentation. Majority of patients had wheeze and Inspiratory crackles on chest examination. Out of 15 patients, 4 patients (26.6\%) were empirically on anti-tubercular treatment at the time of presentation. A total of 6 patients $(40 \%)$ were misdiagnosed and already treated as pulmonary Tuberculosis. Ten patients were treated as cases of refractory asthma before presenting to our OPD.

Majority of patients (73.3\%) had absolute eosinophil count more than 1000 at the time of presentation. All patient had significantly elevated ( $>1000$ ) Total serum lgE level. Similarly all the 15 patients had positive Aspergillus specific IgE.

The findings of chest $x$-ray and high resolution computed tomography (HRCT) scan of chest are highlighted in Table no. 2. Central bronchiectasis (Fig.1) was present in 12 patients (80\%). Mucus plugs and centrilobular nodules were present in $60 \%$ and $66.6 \%$ of patients respectively.

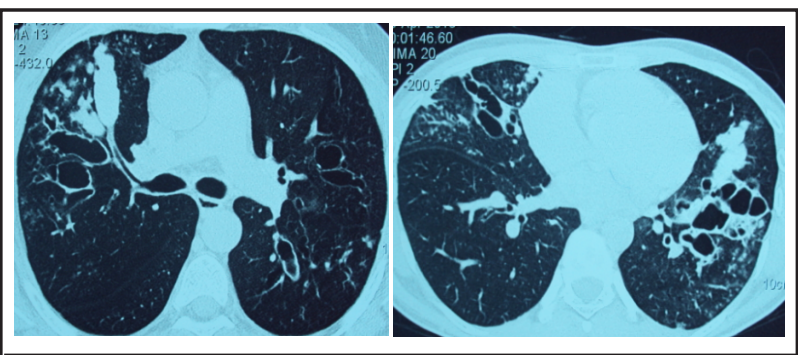

Figure 1. HRCT chest showing central bronchiectasis with mucus plugging and chest $x$ ray showing lung infiltrates in patients with ABPA

All 15 patients required treatment with bronchodilator and systemic steroid. Itraconazole was required in three patients $(20 \%)$ due to frequent relapse. On follow up 14 out of 15 patients i.e. $93.3 \%$ of patients had some radiological clearing of the pulmonary opacity. Total serum IgE decline was more than $50 \%$ in 13 patients (86.6\%). Out of 15 enrolled patients 12 patients (80\%) underwent remission with the treatment whereas three patients had frequent relapses in their clinical course. Treatment and outcomes are highlighted in Table no. 3.

\begin{tabular}{|lll|}
\hline \multicolumn{3}{|l|}{ Table 1. Clinical characteristics of patients. } \\
\hline Clinical features & No. of patients (n) & (\%) \\
Fever & 10 & $66 \%$ \\
Cough & 15 & $100 \%$ \\
Expectoration & 14 & $93.3 \%$ \\
Brownish sputum & 12 & $80 \%$ \\
Hemoptysis & 8 & $53.3 \%$ \\
Weight loss & 8 & $53.3 \%$
\end{tabular}

\begin{tabular}{|lll|} 
Allergic Rhinitis & 9 & $60 \%$ \\
Wheeze & 12 & $80 \%$ \\
Crackle & 11 & $73.3 \%$ \\
Clubbing & 3 & $20 \%$ \\
On ATT & 4 & $26.6 \%$ \\
\hline
\end{tabular}

\begin{tabular}{|lll|}
\hline \multicolumn{3}{|l|}{ Table 2. Radiological features. } \\
\hline Radiological & No. of Patients & Percentage \\
Abnormality & (n) & (\%) \\
Consolidation (x-ray) & 4 & $26.6 \%$ \\
Collapse (x-ray) & 1 & $6.0 \%$ \\
$\begin{array}{l}\text { Opacity (x-ray) } \\
\text { Central }\end{array}$ & 15 & $100 \%$ \\
$\begin{array}{l}\text { Bronchiectasis } \\
\text { (HRCT) }\end{array}$ & 12 & $80 \%$ \\
$\begin{array}{l}\text { Mucus plug (HRCT) } \\
\text { Centrilobular Nodule } \\
\text { (HRCT) }\end{array}$ & 9 & $60 \%$ \\
\hline
\end{tabular}

\begin{tabular}{|c|c|c|}
\hline Treatment and outcome & $\begin{array}{l}\text { No. of } \\
\text { patients (n) }\end{array}$ & $\begin{array}{l}\text { Percentage } \\
(\%)\end{array}$ \\
\hline On Bronchodilator & 15 & $100 \%$ \\
\hline On systemic steroid & 15 & $100 \%$ \\
\hline Itraconazole & 3 & $20 \%$ \\
\hline $\begin{array}{l}\text { Radiological clearance } \\
\text { of opacity }\end{array}$ & 14 & $93.3 \%$ \\
\hline $\begin{array}{l}\text { IgE decline more than } \\
50 \%\end{array}$ & 13 & $86.6 \%$ \\
\hline Remission & 12 & $80 \%$ \\
\hline Frequent relapse & 3 & $20 \%$ \\
\hline
\end{tabular}

\section{DISCUSSION}

ABPA is an allergic pulmonary disorder caused by hypersensitivity to Aspergillus fumigatus clinically manifesting as chronic asthma, recurrent pulmonary infiltrates, fever, brownish expectoration and bronchiectasis. ${ }^{3,6}$

The problem is not well recognized in Nepal. There is only one case report published by one of the investigators of this study. ${ }^{7}$

The Rosenberg-Patterson criteria form the basis of a diagnosis of ABPA, with the presence of six of the following criteria making the diagnosis of ABPA almost certain: presence of asthma, current or previous infiltrates on chest radiograph or CT, immediate cutaneous reactivity to Aspergillus using a skin-prick test or intradermal injection, elevated total serum IgE 
Agarwal et al. Allergic BronchoPulmonary Aspergillosis in Nepal

concentration (cutoff of $417 \mathrm{IU} / \mathrm{mL}$ is often used), serum precipitating antibodies to A. fumigatus, central bronchiectasis, peripheral blood eosinophilia $(>1.0 \times 109 / \mathrm{L})$, and elevated serum $\operatorname{lgE}$ and/or $\lg G$ to A. fumigatus. ${ }^{5,8-10}$ Minor criteria include finding of Aspergillus in sputum, expectoration of brown mucus plugs, and a delayed skin reaction to Aspergillus antigen (type III). ${ }^{1}$

Despite the description of ABPA as early as 1952 by Hinson and colleagues and in 1972 in India by Shah et al, this disease is still under recognized. This series represents the largest reported case series on ABPA from Nepal. In our case series the proportions of males was higher as compared with females ( $80 \%$ vs. $20 \%)$. This finding was consistent with the two largest case series published from India by Chakrabarti et al and Agrawal et al respectively. ${ }^{9,10}$

The mean age of patient in our study group was 33.06 \pm 9.2 years. The reported mean age group in two largest case series on ABPA from India is 34.4 and 32 years respectively. ${ }^{9-11}$ The most common symptoms in our group of patients were cough, production of sputum, brownish coloured sputum and hemoptysis. The reported incidence of hemoptysis in ABPA is $44.4 \%$ and production of brownish coloured sputum is $47.6 \%$ by Agrawal et al in $2006^{10}$ and $2007 .{ }^{8}$ In other large case series, hemoptysis was observed in 37 to $69 \%$ of patients with ABPA. ${ }^{11}, 12$ Fever is another common clinical presentation of ABPA; fever was present in $66 \%$ of our patients. Fever has been reported in $80 \%$ of patients with ABPA. ${ }^{12}$

ABPA is often misdiagnosed as pulmonary Tuberculosis due to its pulmonary symptoms. Presence of cough and hemoptysis together with fever, pulmonary infiltrates and other constitutional symptoms often leads to misdiagnosis of this condition as a case of pulmonary Tuberculosis. This was also seen in our case series. This problem has been highlighted in other previous case series on ABPA. Agrawal et al, in their large case series on ABPA reported that $46.8 \%$ of the patients had history of treatment for pulmonary Tuberculosis. ${ }^{10}$
Similarly, Chakrbarti et al and Shah et al from India reported that $29 \%$ and $81 \%$ of their patient had history of treatment for Tuberculosis respectively. ${ }^{9,} 11$ In laboratory investigations the most useful tests were Serum IgE level, which was elevated in $100 \%$ of our patients. Aspergillus specific IgE level was also high in $100 \%$ of our patients. Similarly, $73.3 \%$ of our patients had absolute eosinophil count more than 1000. This finding was consistent with case series published by Kumar and Gaur in 2000. They found that $100 \%$ of their patients had elevated serum IgE level and positive Aspergillus specific IgE level respectively. ${ }^{13}$ Central bronchiectasis has been reported in $73.1 \%$ of patients with ABPA. ${ }^{10}$ Different case series on ABPA have reported the presence of central bronchiectasis in 69 to $99 \%$ of patients. ${ }^{11-15}$

All of our patients were treated with inhaled bronchodilator and oral glucocorticoid. $80 \%$ of our patients underwent long term remission on subsequent follow up whereas $20 \%$ had one or more relapses of disease. The patients, who had relapse, were treated with antifungal Itraconazole besides systemic corticosteroids. Rosenberg et al in 1977 in their case series on ABPA with 20 patients, reported $100 \%$ remission rate after treatment with corticosteroid alone. ${ }^{15}$ Agrawal et al in 2006 reported $20 \%$ relapse rate of $A B P A$ in their case series of 126 patients with ABPA. ${ }^{10}$ The treatment outcome in our group of patients was consistent with other case series as described previously.

\section{CONCLUSIONS}

ABPA is an under recognized clinical condition in Nepal. Due to high prevalence of Pulmonary Tuberculosis and Bronchial Asthma, ABPA is often misdiagnosed with these clinical conditions. A high index of clinical suspicion and relevant investigations leads to the diagnosis of ABPA. Once diagnosed, treatment with systemic steroids and inhaled bronchodilators leads to excellent clinical outcome.

\section{REFERENCES}

1. Agarwal R. Allergic bronchopulmonary aspergillosis. Chest. 2009; 135: 805-26.

2. Hinson KFW, Moon AJ, Plummer NS. Broncho-pulmonary aspergillosis; a review and a report of eight new cases. Thorax. 1952; 7:317-33.

3. Agarwal R, Chakrabarti A. Epidemiology of allergic bronchopulmonary aspergillosis. In: Pasqualotto AC, editor. Aspergillosis: From Diagnosis to Prevention. New York: Springer. 2010; p 671-88.
4. Hogan C, Denning DW. Allergic bronchopulmonary aspergillosis and related allergic syndromes. Semin Respir Crit Care Med. 2011; 32: 682-92.

5. Rosenberg M, Patterson R, Mintzer R, Cooper BJ, Roberts $\mathrm{M}$. Clinical and immunologic criteria for the diagnosis of allergic bronchopulmonary aspergillosis. Ann Intern Med. 1977; 86: 405-14. 
6. Greenberger PA, Patterson R, Ghory A. Late sequelae of allergicbronchopulmonary aspergillosis. J Allergy Clin Immunol.1980; 66:327-35.

7. Chokhani R, Neupane S, Kandel IS. Allergic bronchopulmonary aspergillosis - a case report. Nepal Med. Col. J. 2004; 6(2):154-6.

8. Agarwal R, Gupta D, Aggarwal AN. Clinical significance of hyperattenuating mucoid impaction in allergic bronchopulmonary aspergillosis: an analysis of 155 patients. Chest. 2007; 132:1183-90.

9. Chakrabarti A, Sethi S, Raman DS. Eight-year study of allergic bronchopulmonary aspergillosis in an Indian teaching hospital. Mycoses. 2002; 45:295-9.

10. Agarwal R, Gupta D, Aggarwal AN. Allergic bronchopulmonary aspergillosis: lessons from 126 patients attending a chest clinic in North India. Chest. 2006; 130:442-8.
11. Shah A, Panchal N, Panjabi C. Allergic bronchopulmonary aspergillosis: a review from India. Allergy Clin Immunol Int. 2003; Suppl 1:104.

12. Shah A. Allergic bronchopulmonary aspergillosis: an Indian perspective. Current Opinion in Pulmonary Medicine. 2007, 13:72-80.

13. Kumar R, GaurSN. Prevalence of allergic bronchopulmonary aspergillosis in patients with bronchial asthma. Asian Pac J Allergy Immunol. 2000; 18:181-5.

14. Behera D, Guleria R, Jindal SK. Allergic bronchopulmonary aspergillosis: a retrospective study of 35 cases. Indian J Chest Dis Allied Sci. 1994; 36:173-9.

15. Rosenberg M, Patterson R, Mintzer R, Cooper BJ, Roberts $\mathrm{M}$, Harris KE. Clinical and immunologic criteria for the diagnosis of allergic bronchopulmonary aspergillosis. Ann Intern Med. 1977; 86: 405-14. 